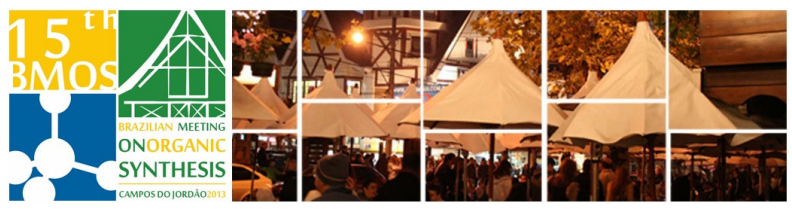

\title{
Theoretical study of the regiospecific synthesis of pyrazole-5- carboxylate from unsymmetrical enaminodiketones
}

\author{
Thiago C. Rozada, Michael J. V. da Silva, Rodrigo M. Pontes, Ernani A. Basso, \\ Fernanda A. Rosa* \\ Universidade Estadual de Maringá, Avenida Colombo, 5790, Zona 07, Maringá - PR, CEP 87020-900 \\ *farosa@uem.br
}

Keywords: Regiospecific Synthesis, Pyrazole, Transition State

\section{INTRODUCTION}

The synthesis of pyrazoles has been the subject of consistent interest because of the wide applications for such heterocycles. ${ }^{1}$ Nevertheless the synthesis of such compounds with unsymmetrical 1,3dicarbonyl substrates often produces a regioisomeric mixture of pyrazoles with generally poor selectivity. However, Rosa and co-workers reported the regiospecific synthesis of pyrazole-5carboxylates from unsymmetrical enaminodiketones. ${ }^{2}$ Thus, considering the application of pyrazoles and the regiospecificity of the reactions presented by Rosa, we conducted a detailed theoretical investigation of the structure of the reagents, products and possible transition states in the reactions (Figure 1).

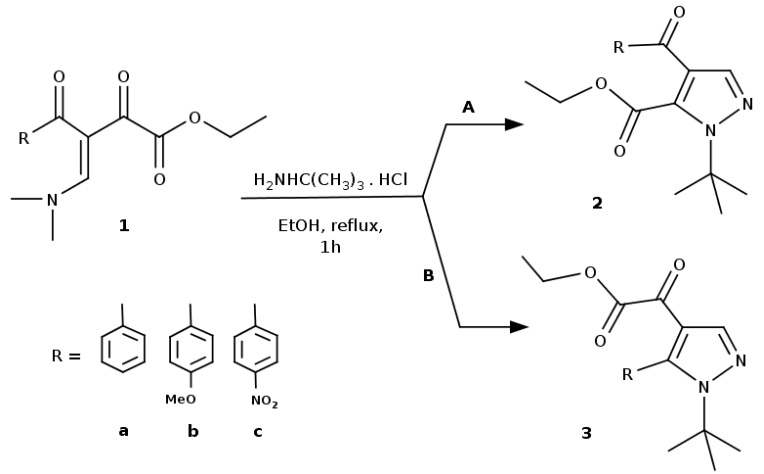

Figure 1. Enaminodiketones (1) and possible pyrazoles (2-3) from reaction with tert-butylhydrazine hydrochloride. ${ }^{2}$

\section{RESULTS AND DISCUSSION}

Geometries of the reactant, transition states and products of the reaction studied were optimized at M06-2X/6-31++G(d,p) level in ethanol (IEF-PCM) using the Gaussian 09. The optimized geometries were characterized as a minimum or transition state and the ZPE energies for all structures were determined (Table 1). All transition states were proved by applying the IRC methodology.

Rosa and co-workers observed the formation of only pyrazole 2 (cyclization A). The study of the transition states for the two cyclizations reveled that compounds 2a-c are the most stable (thermodynamic products). Whereas for cyclization $\mathrm{B}, \mathbf{1 a - c}$ are the more stable structures.
Table 1. Relative energies $\left(\mathrm{kcal} \mathrm{mol}^{-1}\right)$ for compounds a-c.

\begin{tabular}{|c|c|c|c|c|c|c|}
\hline & \multicolumn{2}{|c|}{$\mathbf{a}$} & \multicolumn{2}{|c|}{ b } & \multicolumn{2}{|c|}{ C } \\
\hline & A & B & A & B & A & B \\
\hline Reac. & 4.55 & 0.00 & 5.37 & 0.00 & 4.93 & 3.03 \\
\hline TS & 14.18 & 9.47 & 14.96 & 10.54 & 10.89 & 10.99 \\
\hline Prod. & 0.90 & 4.17 & 2.17 & 5.70 & 0.00 & 3.64 \\
\hline$\Delta \mathrm{E}^{\neq}$ & 9.64 & 9.47 & 9.59 & 10.54 & 5.95 & 7.95 \\
\hline
\end{tabular}

For $\mathbf{a}$ and $\mathbf{b}$, the TSB was more stable than TSA. For $\mathbf{c}$ both TSs have similar energies. The activation energies for $\mathbf{2 a}$ and $\mathbf{3 a}$ are very close and the $\mathrm{OCH}_{3}$ introduction in the aromatic ring raised the activation energies of $\mathbf{3 b}$ (cyclization $\mathrm{B}$ ) when compared with 3a but doesn't give much difference for cyclization A. On the other hand, the $\mathrm{NO}_{2}$ introduction in the aromatic ring gives the smallest activation energies for both cyclizations.

The relative stability of TSB over TSA for $\mathbf{a}$ and $\mathbf{b}$ could be explained by the orientation of the TSB that permit a hydrogen bond between the protonated carbonyl with the ester carbonyl. For the TSA, this kind of interaction was observed only to compound $\mathbf{c}$ and helps to explain the higher stability of this TS. NBO analyzes showed that the $\mathrm{LP}_{\mathrm{O}} \rightarrow \delta^{*} \mathrm{O}-\mathrm{H}$ for compound a was only $4.08 \mathrm{kcal} \mathrm{mol}^{-1}$ in TSA but $33.87 \mathrm{kcal} \mathrm{mol}^{-1}$ for TSB. The energy difference between 2a-c and 3a-c could be associated with the higher hyperconjugative orbital interactions in $\mathbf{2 a - c}$ due to the presence of a carbonyl between the aromatic ring and the pyrazole.

\section{CONCLUSION}

The regioespecific formation of the pyrazoles $\mathbf{2 a - c}$ (cyclization A) could be explained with the thermodynamic data once these products are more stable that the pyrazoles 3a-c.

ACKNOWLEDGEMENTS
CNPq - Fundação Araucária - PQU/UEM
REFERENCES

${ }^{1}$ Katritzky, A. R.; Wang, M.; Zhang, S.; Voronkov, M. V. J. Org. Chem.
${ }_{2}^{2001,66,6787 . ~}$
Mosa, F. A.; Machado, P.; Vargas, P. S.; Bonacorso, H. G., Zanatta, N.;
Martins, M. A. P. Synlett 2008, 11, 1673.

